\title{
DELIVERY TEMPORAL PATTERNS IN INFANTS WITH PERINATAL HYPOXIC-ISCHEMIC
} ENCEPHALOPATHY (HIE)

\author{
A. Garcia-Alix ${ }^{1}$, G. Arca $^{2}$, V. Cortes ${ }^{1}$, F. Gaya ${ }^{3}$, L. Perez ${ }^{4}$, J. Diaz ${ }^{4}$, E. Valverde ${ }^{5}$ \\ ${ }^{1}$ Pediatrics, Hospital Sant Joan de Deu, Universidad de Barcelona, ${ }^{2}$ Pediatrics, Hospital Clinic-Maternitat. \\ Universidad de Barcelona, Barcelona, ${ }^{3}$ Hospital La Paz, ${ }^{4}$ Hospital La Paz, Autonoma University, \\ ${ }^{5}$ Pediatrics, Hospital La Paz, Autonoma University, Madrid, Spain
}

Background: Delivery temporal patterns of infants with perinatal EHI may indicate variations in care provided to women in labor, as well as potential for prevention, systems improvement and the burden of work, but those patterns have been scarcely investigated.

Objective: To examine:

1) temporal patterns in deliveries of infants with HIE: day of week, weekday/weekend, month and season.

2) Whether the timing influences the severity of HIE.

Design: Retrospective cohort study of 90,954 newborns born at a University tertiary Hospital between 20002009. During this period, 109 newborns $>34$ w GA had perinatal HIE.

Results: The incidence of HIE was 1.088 per 1000 live births, and 0.49 per 1000 for moderate and severe HIE. The incidence showed a downward linear trend throughout the study period ( $\mathrm{s}=-5.37 ; \mathrm{P}<0.05)$. We did not find an impact of the month, day of the week or season on the risk of delivering an infant with perinatal HIE, even after adjusting for year and month. When the weekday/weekend impact was examined, we found a higher risk for weekday deliveries $(\mathrm{p}<0.0008)$. This association remained significant after adjusting for year and month, and nearly reached statistical significance for infants with moderate and severe HIE $(\mathrm{p}=0.085)$.

Conclusion: Temporal patterns including day of the week, month, and season do not affect the risk of delivering an infant with HIE in a University Hospital in Spain. In contrast to population based studies, we found a higher risk of HIE for deliveries occurring in working days. 